Journal of

Applied Crystallography

ISSN 0021-8898

Received 15 January 2002

Accepted 9 September 2002

C) 2003 International Union of Crystallography Printed in Great Britain - all rights reserved

\section{Simultaneous light and small-angle neutron scattering on aggregating concentrated colloidal suspensions}

\author{
Sara Romer, ${ }^{\text {a,b }}$ Claus Urban, ${ }^{\mathrm{c}}$ Vladimir Lobaskin, ${ }^{\mathrm{a}}$ Frank Scheffold, ${ }^{\mathrm{a}}$ Anna \\ Stradner, ${ }^{a}$ Joachim Kohlbrecher ${ }^{\mathrm{d}}$ and Peter Schurtenberger ${ }^{\mathrm{a} *}$

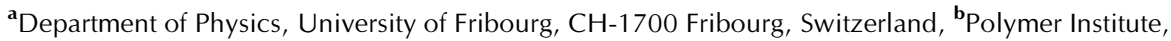 \\ ETH Zürich, CH-8092 Zürich, Switzerland, ' $\mathrm{CS}$ Instruments, c/o Department of Physics, University \\ of Fribourg, $\mathrm{CH}-1700$ Fribourg, Switzerland, and ${ }^{\mathrm{d} P a u l}$ Scherrer Institute, $\mathrm{CH}-5232$ Villigen, \\ Switzerland. Correspondence e-mail: peter.schurtenberger@unifr.ch
}

\begin{abstract}
A new sample environment has been developed in order to perform light and small-angle neutron scattering (SANS) simultaneously on colloidal systems. The combination of SANS and diffusing wave spectroscopy (DWS) is of particular use in the high-concentration regime. DWS provides information on the local dynamic properties of the individual particles, whereas SANS gives access to the structural properties on similar length scales. The combination of both methods thus allows one to obtain structural and dynamic information over a very large range of length and time scales. Using this new setup, the onset of aggregation and the sol-gel transition in concentrated destabilized polystyrene sphere suspensions have been investigated. At the gel point, a dramatic change of the particle dynamics from diffusion to a subdiffusive arrested motion is observed. However, while the DWS measurements indicate that dramatic changes in the local dynamics occur over a long period, the SANS pattern quickly reaches its final appearance. The SANS experiments thus indicate that a fluid-like structure is arrested in the course of the gel formation. The data are found to be in good qualitative agreement with computer simulations.
\end{abstract}

\section{Introduction}

Concentrated colloidal gels are fascinating systems. On the one hand, they are ideal models for the study of the elastic properties of highly disordered networks. On the other hand, sol-gel processes are commonly used in colloid technology and engineering, as for instance in ceramics processing (Gauckler et al., 1999). The structure and dynamics of gels have been the subject of intensive theoretical and experimental activity in recent years. In particular, scattering techniques are ideal tools for the study of such systems. Previous studies on concentrated highly charged colloidal suspensions have shown that diffusing wave spectroscopy (DWS) can be used to follow the sol-gel transition in dense systems (Romer et al., 2000). DWS extends the classical dynamic light scattering (DLS) to the limit of very strongly scattering media (Maret \& Wolf, 1987; Pine et al., 1988). In such a limit, the propagation of light through the system can be described by a diffusion equation and it is thus possible to determine quantitatively the intensity autocorrelation function $g_{2}(\tau)=$ $\langle I(t) I(t+\tau)\rangle /\left\langle|I(t)|^{2}\right\rangle$ and the average mean-square displacement of the single scatterers on length scales much smaller than the particle size. Although DWS allows the particle dynamics to be determined experimentally, it gives only little information about the sample structure. As a consequence, other scattering methods need to be considered.
The combination of SANS with DWS is of particular use in the high-concentration regime. DWS is a powerful tool to obtain information about the local dynamical properties, i.e. the mean-square displacement of the individual particles. It probes particle motion on very short length scales and can, for example, measure motions of particles of the order of $1 \mu \mathrm{m}$ in diameter on length scales of less than $1 \mathrm{~nm}$. SANS on the other hand gives access to structural properties of dilute and concentrated systems on similar length scales. Therefore, DWS and SANS provide important complimentary information that cannot be obtained otherwise. In the present article we report on a new sample environment which allows time-resolved DWS and small-angle neutron scattering (SANS) to be performed simultaneously on gelling systems. In particular, we follow the destabilization process in concentrated chargestabilized suspensions of polystyrene spheres. With this new setup, we can quantitatively monitor the changes in the sample structure and dynamics during the sol-gel transition.

\section{Experimental section}

The samples consist of solutions of monodisperse polystyrene spheres (sulfate latex particles, Interfacial Dynamics Corporation), at volume fractions up to $26 \%$. Aggregation and gelation are induced by screening the electrostatic interactions 
with salt. However, for highly concentrated samples, it is very difficult to achieve a homogeneous reproducible destabilization by simply adding salt. Recently a novel method based on an in situ variation of the ionic strength has been introduced (Gauckler et al., 1999). The destabilization of the colloidal suspensions is induced with an internal chemical reaction, which allows the slow production of ions (the urease catalysed hydrolysis of urea). This can be extended easily to any electrostatically stabilized suspension, such as polystyrene particles (Romer et al., 2000).

The small-angle neutron scattering measurements were carried out at the SANS instrument at the Paul Scherrer Institute (PSI) in Villigen, Switzerland. For the SANS experiments we used a neutron wavelength of $1.68 \mathrm{~nm}$ and a detector distance of $18 \mathrm{~m}$, which corresponds to a $q$ range of $0.01-0.1 \mathrm{~nm}^{-1}$. Multiple neutron scattering is suppressed by partially contrast matching the colloidal particles using a water-heavy water mixture (40:60). Small additional contrast corrections were performed for each sample individually by adding water (or heavy water) in order to guarantee the absence of multiple neutron scattering. However, this solvent composition does not affect the light scattering contrast and the systems show strong multiple light scattering. In order to perform light and neutron scattering simultaneously, we have modified the standard sample environment. The basic sample support consists of an $x y z$ and rotation table, where various sample environments can be accommodated. On that table, we have mounted an additional aluminium plate $(705 \times 900 \times$ $10 \mathrm{~mm}$ ), to which the optical devices and the samples can be fixed. We have used a sample holder for commercial spectroscopy cuvettes (path length $1 \mathrm{~mm}$ ) which can be temperature controlled. A schematical top view of the modified sample environment is presented in Fig. 1. For the light scattering, an $\mathrm{He}-\mathrm{Ne}$ laser (wavelength $\lambda=633 \mathrm{~nm}$, Uniphase, $15 \mathrm{~mW}$ ) is employed. The beam is reflected by a mirror

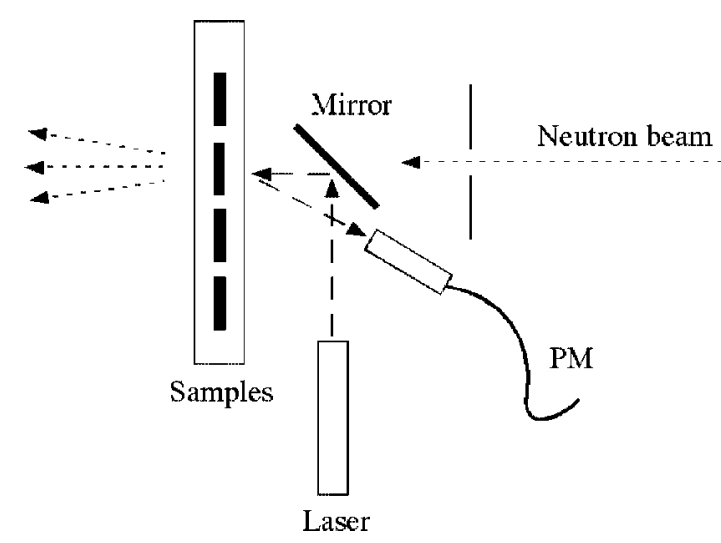

Figure 1

Schematic top view of the setup for simultaneous light and small-angle neutron scattering. An He-Ne laser beam is reflected by a mirror and illuminates a sample located in a multi-sample cell holder. The mirror is positioned in the incoming neutron beam at an angle of $45^{\circ}$. The multiply scattered light is then collected in backscattering geometry with a single mode fibre, then guided to two photomultipliers (PM) and subsequently analysed with a digital correlator employing a pseudo cross-correlation scheme. positioned at an angle of $45^{\circ}$ in the incoming neutron beam and illuminates the sample. As a mirror, we use a silica wafer that has been coated with a $10 \mathrm{~nm}$ aluminium layer in order to improve its light reflectivity but at the same time avoid undesirable neutron scattering. The multiply scattered light is then collected in backscattering geometry with a single mode fibre. The signal is then split and fed into two photomultipliers (Type H5783P-01, Hamamatsu, Japan) and subsequently analysed with a digital correlator (Flex99R-FCS-12, Correlator Corp.) that performs a pseudo cross-correlation analysis. The DWS backscattering geometry is very convenient because it requires access to a sample from one side only. However, the setup described above allows also different detection angles in the range of about $40-175^{\circ}$. The light scattering measurements are therefore not restricted only to the multiple-scattering regime, but they can be extended to the classical dynamic light scattering technique.

For the SANS experiments the samples were kept in stoppered quartz cells (Hellma, Germany) with a path length of $1 \mathrm{~mm}$. The neutron spectra of water were also measured in a $1 \mathrm{~mm}$ path-length quartz cell. The raw spectra were corrected for background from the solvent, sample cell and electronic noise by conventional procedures. Furthermore, the twodimensional isotropic scattering spectra were azimuthally averaged, converted to absolute scale, and corrected for detector efficiency by dividing by the incoherent scattering spectrum of pure water (Cotton, 1991; Jacrot \& Zaccai, 1981; Ragnetti \& Oberthür, 1986; Wignall \& Bates, 1987).

The smearing induced by the instrumental setup is included in the data analysis discussed below. The ideal model scat-

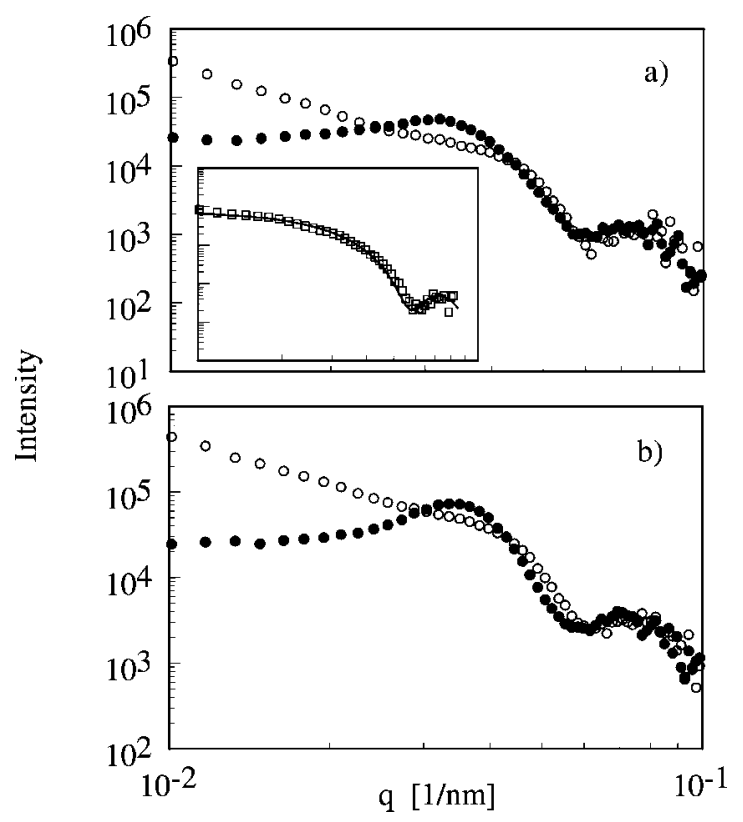

Figure 2

The $q$ dependence of the SANS intensity curves for the stable system (filled symbols) and for the final gel (open symbols): (a) 19\% sample; $(b)$ $26 \%$ sample. Inset: fit of the form factor $P(q)$ for a $0.8 \%$ dispersion (radius $=80 \mathrm{~nm}$, polydispersity $=6 \%$ ) using a form factor of polydisperse spheres. 
tering curves were smeared by the appropriate resolution function when the model scattering intensity was compared with the measured one by means of least-squares methods (Pedersen et al., 1990; Barker \& Pedersen, 1995). The parameters in the models were optimized by conventional leastsquares analysis, and the errors of the parameters were calculated by conventional methods (Bevington, 1969).

\section{Results and discussion}

Fig. 2 shows neutron scattering curves for the stable system and the final gel, after $24 \mathrm{~h}$, for two different volume fractions $(19 \%$ and $26 \%)$. The measured intensity as a function of the scattering vector $q$ contains contributions from the particle structure [particle form factor $P(q)$ ] as well as from interparticle interaction effects [structure factor $S(q)$ ]. $P(q)$ can be determined experimentally from a dilute suspension. We have used a $0.8 \%$ dispersion and the result has been fitted using a polydisperse form factor convoluted with the instrumental resolution (see inset of Fig. 2). On that basis we determine a particle radius of $80 \mathrm{~nm}$ and a polydispersity of about $6 \%$, in good agreement with supplier specifications $(170 \mathrm{~nm}$, polydispersity $4 \%$ ) and previously reported SANS and light scattering data (Rojas-Ochoa et al., 2002). Measurements of the gels at higher $q$ ( $6 \mathrm{~m}$ detector distance) where contributions from $S(q)$ do not need to be taken into account, show that $P(q)$ does not change during the destabilization process. This shows that no measurable fusion processes of the single polystyrene particles take place during our measurements and that the change of the $q$ dependence of the intensity is only due to cluster and gel formation. For both gels, the structurefactor peak disappears and an enormous intensity increase at low $q$ occurs due to the long-range correlations as soon as the ionic strength increases. SANS measurements on concentrated colloidal silica gels have shown a similar behaviour (Muzny et al., 1994).

To obtain complementary information on the microscopic spatial structure of the suspension, we performed Monte Carlo

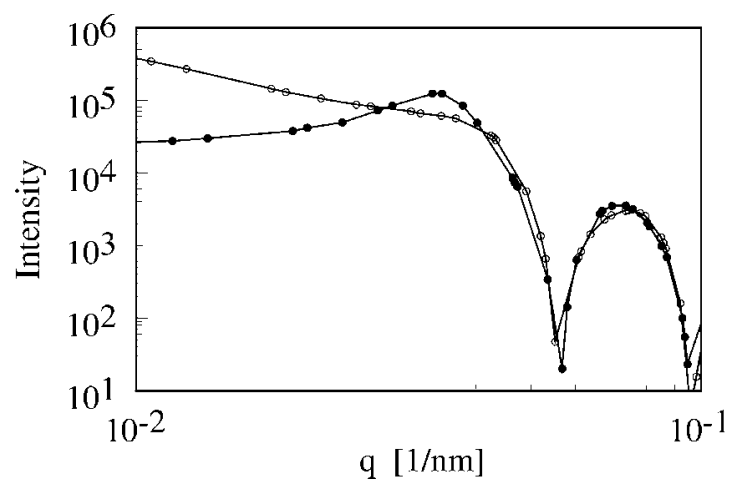

Figure 3

Scattered intensity as a function of the scattering vector as predicted by Monte Carlo simulation for the stable suspension (filled symbols) and for the final gel (open symbols). The parameters of the DLVO-type interaction potential were estimated by taking into account the concentration of the salt produced in the enzymatic reaction at different stages of gelation (see text for details). simulations of the spherical particles interacting through a DLVO potential (Russel et al., 1989; Evilevitch et al., 2001). The interaction potential comprises van der Waals attraction expressed by the Hamaker formula for spheres, electrostatic repulsion in the Debye-Hückel form, and a hard-core potential preventing interpenetration of the particles. The particle charge was estimated from the surface charge density, assuming full dissociation of surface charges. The DebyeHückel screening length was estimated taking into account the concentration of dissociated charges and concentration of the salt produced in the enzymatic reaction at different stages of
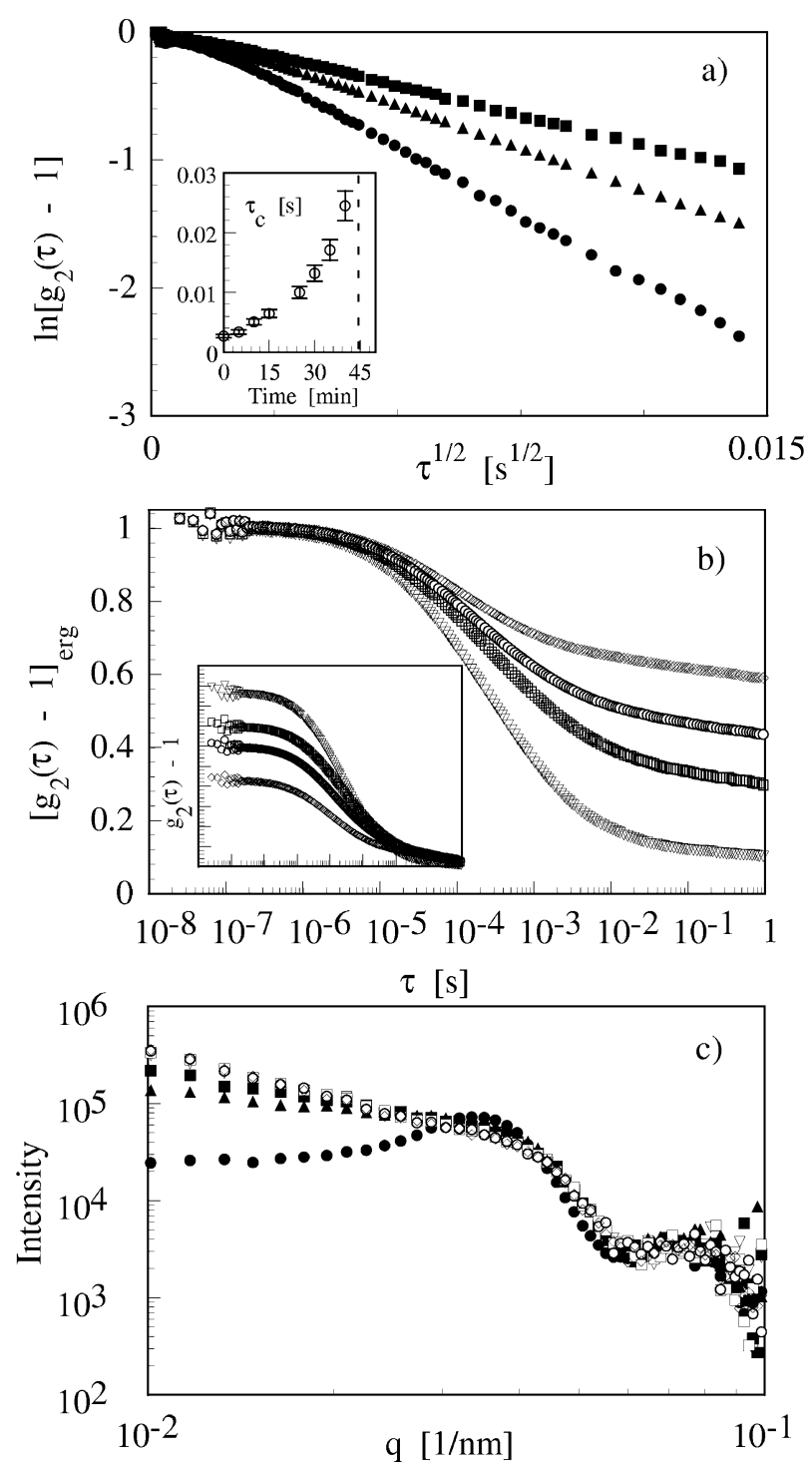

Figure 4

Time evolution of the DWS autocorrelation functions and of the $q$ dependence of the scattered intensity of a $26 \%$ particle suspension undergoing a sol-gel transition. (a) Stable suspension at $t=0 \mathrm{~min}$ and time evolution after destabilization for $t=15$ and $30 \mathrm{~min}$ before the gel point (from bottom to top). Inset: time evolution of the characteristic time $\tau_{c}$. $(b)$ Correlation functions $\left[g_{2}(\tau)-1\right]_{\mathrm{erg}}$ in the gel state for $t=55$, 80,105 and $210 \mathrm{~min}$ (from bottom to top). Inset: unshifted correlation functions for $t=55,80,105$ and $210 \mathrm{~min}$ (from top to bottom). (c) SANS intensity curves [same symbols correspond to same times as in $(a)$ and $(b)]$. 
gelation. The particle hard-core diameter was set to $170 \mathrm{~nm}$, the Hamaker constant to a typical value for polystyrene $(1.3 \times$ $10^{-20} \mathrm{~J}$ ), and the screening length was varied from $30 \mathrm{~nm}$ at low salt concentration to $1.8 \mathrm{~nm}$ at the highest salt concentration. We used an ensemble of 100 particles at volume fraction 0.1. As shown in Fig. 3, the $q$ dependence of the intensity for the stable suspension and for the final gel found in the simulations are in good qualitative agreement with the experimentally observed structures. The simulation shows that percolating clusters are formed and the disordered structure is frozen immediately once the salt concentration reaches the amount needed to screen the stabilizing electrostatic repulsion and initiate the aggregation in the primary potential minimum.

Further studies of the gel formation were then performed by using time-resolved DWS and SANS measurements. We followed the destabilization of a $26 \%$ sample. To destabilize the system we added urease at a temperature $T$ of approximately $276-278 \mathrm{~K}$, at which its activity is sufficiently reduced. The urea represents $10 \%$ of the solvent volume. The urease concentration ( 75 units per $1 \mathrm{ml}$ of $\mathrm{H}_{2} \mathrm{O}$ and $\mathrm{D}_{2} \mathrm{O}$ ) was chosen such that the aggregation and gelation at the selected temperature of $298 \mathrm{~K}$ takes place slowly. Fig. 4 displays typical intensity autocorrelation functions $g_{2}(\tau)-1$ during the coagulation, where $\tau$ is the correlation time. For an optically dense sample (thickness $L \gg l^{*}$, where $l^{*}$ is the photon transport mean path) the expression of the intensity autocorrelation function for the DWS backscattering geometry is (Weitz \& Pine, 1993)

$$
g_{2}(\tau)-1=\exp \left\{-2 \gamma\left[k^{2}\left\langle\Delta r^{2}(\tau)\right\rangle\right]^{1 / 2}\right\},
$$

where $k$ is the wavenumber of the light in the medium and $\left\langle\Delta r^{2}(\tau)\right\rangle$ is the single-particle mean-square displacement. The geometrical factor $\gamma$ depends slightly on both polarization and on the anisotropy of the scattering. Its average value, $\langle\gamma\rangle$, is about 2. For a stable system in which the particles undergo Brownian diffusion, the autocorrelation function can be simplified to

$$
g_{2}(\tau)-1=\exp \left[-2 \gamma\left(6 \tau / \tau_{c}\right)^{1 / 2}\right] .
$$

The characteristic time $\tau_{c}$ is directly related to the effective diffusion coefficient of the particles $D_{\text {eff }}=\left\langle\Delta r^{2}(\tau)\right\rangle / 6 \tau$ as $\tau_{c}=$ $1 /\left(k^{2} D_{\text {eff }}\right)$. At long times, the autocorrelation function completely decorrelates. For gels, on the contrary, the autocorrelation function shows a plateau (Krall \& Weitz, 1998; Romer et al., 2000). In a gel the particles can no longer diffuse, but they undergo a constrained motion which is characterized at long times by a maximum displacement $\left\langle\Delta r^{2}(\tau)\right\rangle_{\tau \rightarrow \infty}=\delta^{2}$. For large correlation times, the autocorrelation function for a gel is then

$$
\left[g_{2}(\tau)-1\right]_{\tau \rightarrow \infty} \simeq \exp \left[-2 \gamma\left(k^{2} \delta^{2}\right)^{1 / 2}\right] .
$$

In the early stages of the coagulation process, we observe that the decay of the autocorrelation function shifts to longer correlation times due to the slower motion of the clusters formed during the aggregation. Fig. 4(a) shows the logarithm of the correlation functions, $\ln \left[g_{2}(\tau)-1\right]$, as a function of $\tau^{1 / 2}$ as aggregation progresses. $1 /\left(\tau_{c}^{1 / 2}\right)$ is then proportional to the linear slope of these curves (inset of Fig. 4a) (Weitz \& Pine, 1993). Near the gel point this characteristic time dramatically increases due to the diverging cluster size. The sol-gel transition occurs when a single cluster fills the entire sample volume (after about $45 \mathrm{~min}$ ). At that point the correlation functions $g_{2}(\tau)-1$ no longer decay to zero, but remain finite within our measurement window, showing the persistence of some correlations for long times. In principle, light scattered from such systems can be expected to consist of a static (timeindependent) part, leading to a plateau in the correlation function, and a fluctuating part. The static part of the scattered intensity signal, on the other hand, depends on the specific position of all particles in the scattering volume and does not reflect the ensemble-averaged light scattering properties of the system. Such a scattering signal is therefore considered to be non-ergodic. Different methods have been proposed to average properly the light scattering signal from non-ergodic media (Xue et al., 1992; Scheffold et al., 2001; Pusey \& van Megen, 1989). For the analysis of our data we follow considerations explained in detail by Pusey \& van Megen (1989). Those authors show that the average over a series of independent measurements of light scattered from different sample configurations yields a proper ensemble average. In that case the ensemble-averaged correlation function can be simply obtained by shifting the intercept of the measured and averaged correlation functions $g_{2}(\tau)-1$ to one (see Fig. $4 b$ ). For our sample we take advantage of the fact that while the system does not relax completely within our measurement window, i.e. less than $1 \mathrm{~s}$, it nevertheless is expected to show a decay on a time scale between seconds and minutes. This can already be seen by the fact that the average intensity varies strongly on this long time scale. Each of our measurements shown in the inset of Fig. 4(b) is therefore an average over ten subsequent individual measurements of $1 \mathrm{~min}$ duration, resulting in reproducible ensemble averages of the measured correlation functions (Fig. 4b). Comparing the data with previous DWS measurements on similar gels, we find that qualitatively the shape and the time evolution of the correlation function is well reproduced, showing a plateau-like behaviour for long correlation times with a plateau value increasing with time (Romer et al., 2000).

The SANS spectra were collected during $15 \mathrm{~min}$ for each data set in order to obtain sufficiently good counting statistics. Fig. 4(c) displays the intensity curves corresponding to the DWS measurements over the entire coagulation process. It can be clearly seen that the peak in the structure factor already disappears in the early pre-gel stages, after the first $15 \mathrm{~min}$. Around and after the gel point, the gel structure no longer changes within our $q$ range, although the dynamics become more and more constrained, as underlined by the increasing plateau in the light scattering data. This finding is also supported by separately performed measurements of the static transmission $T$ of a light beam through the gelling samples. In light scattering experiments performed in concentrated systems, the photons can be treated as random walkers and in this approximation the distance that they must travel to randomize their direction is the so-called transport 
mean free path $l^{*}$. For scatterer sizes close to or larger than the photon wavelength, the scattering process is no longer isotropic and several scattering processes are required for a complete randomization. Therefore $l^{*}$ is larger than the mean distance between scattering events. The microscopic parameter $l^{*}$ is a measure of the sample microstructure since it depends on the individual particle properties [size, shape, refractive index $n$; i.e. $P(q)]$ as well as on the interparticle correlations and local density $[S(q)]$ (Weitz \& Pine, 1993):

$$
1 / l^{*} \simeq \int_{0}^{4 \pi n / \lambda} P(q) S(q) q^{3} \mathrm{~d} q .
$$

Thus $l^{*}$ contains the same information as the SANS intensity curves, although the structural information appears only in the form of an integral. Therefore, changes in $l^{*}$ are related to changes in the sample microstructure. Experimentally, $l^{*}$ can be directly determined from the transmitted light intensity since $l^{*} \simeq T$. Fig. 5 displays the typical time evolution of $l^{*}$ for a $19 \%$ sample compared with its gel point, which has been determined with high accuracy from DWS measurements in transmission using a different sample (dashed line in Fig. 5) (Rojas-Ochoa et al., 2002; Kaplan et al., 1994). For the destabilized suspension, an increase in $l^{*}$ is observed only for the early stages of aggregation, while later, near the gel point and in the gel state, the value of $l *$ can be considered constant. This confirms our picture of a local structure that no longer changes in the later stages of the gelation process.

For the $19 \%$ suspension, we can quantitatively compare the values of $l^{*}$ that have been experimentally determined from light transmission with the values that can be calculated using equation (4) and the SANS results. While in light scattering the form factor has to be described using Mie theory (van de Hulst, 1981; Bohren \& Huffman, 1983), the effects of interparticle interactions can be appropriately described by the structure factor obtained from neutron scattering, $S(q)_{\text {SANs. }}$. We have determined the structure factors for the stable and coagulated $19 \%$ samples by dividing the total (concentrationnormalized) SANS intensity at that volume fraction by the scattering spectrum of the dilute system which describes the particle form factor for neutron scattering, $I(q)_{19 \%} / I(q)_{0.8 \%} \simeq$

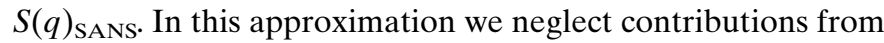

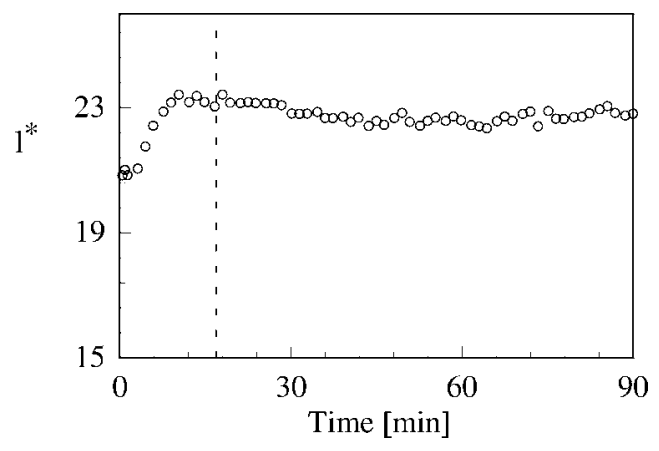

Figure 5

Time evolution of $l^{*}$ as determined from static transmission measurements [gel point (dashed line)]. For this measurement we used a higher urease concentration. the experimental resolution. However, for our experimental $18 \mathrm{~m}$ setting, the resolution effects are small. From equation (4), with $P(q) S(q)=P(q)_{\mathrm{Mie}} S(q)_{\mathrm{SANS}}$, we find the normalized transport mean free path $l_{\mathrm{gel}}^{*} / l_{\text {stable }}^{*}=1.09$, which compares well with the value from the light transmission, $l_{\mathrm{gel}}^{*} / l_{\text {stable }}^{*}=1.08$.

Our experimental results and computer simulations demonstrate that for our colloidal suspensions the sol-gel transition is a frozen-in process. In the gel state the particles undergo only limited displacements, which become more constrained with time. As a consequence, the non-ergodicity in the DWS signal increases with ageing, while the $q$ dependence of the SANS intensity remains constant. This is due to a stiffening of the network (increasing network spring constant) and is not due to a structural evolution.

The combination of neutron and light scattering provides simultaneous information about the structure and the dynamics of time-dependent complex systems, such as our gelling suspensions. In particular, the combination of SANS with multiple light scattering has the advantage that DWS probes particle motion on length scales comparable with that of SANS (1-100 nm). In order to obtain quantitative DWS measurements in the gel state, the setup will have to be improved to allow for measurements in transmission geometry and/or using a CCD camera instead of a photomultiplier in order to perform multi-speckles correlation analysis (Kirsch et al., 1996; Cipelletti et al., 2000; Cardinaux et al., 2002).

We kindly acknowledge Markus Stieger, University of Kiel (Germany), who provided us with the polystyrene particles. This work was supported by the Swiss National Science Foundation (grant No. 21-57141.99).

\section{References}

Barker, J. G. \& Pedersen, J. S. (1995). J. Appl. Cryst. 28, 105-114. Bevington, P. R. (1969). Data Reduction and Error Analysis for the Physical Sciences. New York: McGraw-Hill.

Bohren, C. F. \& Huffman, D. R. (1983). Absorption and Scattering of Light by Small Particles. New York: John Wiley.

Cardinaux, F., Cipelletti, L., Scheffold, F. \& Schurtenberger, P. (2002). Europhys. Lett. 57, 738-744..

Cipelletti, L., Manley, S., Ball, R. C. \& Weitz, D. A. (2000). Phys. Rev. Lett. 84, 2275-2279.

Cotton, J. P. (1991). Initial Data Treatment, in Neutron, X-ray and Light Scattering: Introduction to an Investigative Tool for Colloidal and Polymeric Systems, edited by P. Lindner \& T. Zemb, pp. 19-31. Amsterdam: North-Holland.

Evilevitch, A. A., Lobaskin, V., Olsson, U., Schurtenberger, P. \& Linse, P. (2001). Langmuir, 17, 1043-1053.

Gauckler, L. J., Graule, T. \& Baader, F. (1999). Mater. Chem. Phys. 2509, 1-25.

Hulst, H. C. van de (1981). Light Scattering by Small Particles. New York: Dover.

Jacrot, B. \& Zaccai, G. (1981). Biopolymers, 20, 2413-2426.

Kaplan P. D., Dinsmore, A. D., Yodh, A. G. \& Pine, D. J. (1994). Phys. Rev. E, 50, 4827-4835.

Kirsch, S., Frenz, V., Schärtl, W., Bartsch, E. \& Sillescu, H. (1996). J. Chem. Phys. 104, 1758-1761.

Krall, A. H. \& Weitz, D. A. (1998). Phys. Rev. Lett. 80, 778-781.

Maret, G. \& Wolf, P. E. (1987). Z. Phys. B, 65, 409-413. 


\section{research papers}

Muzny, C. D., Straty, G. C. \& Hanley, H. J. M. (1994). Phys. Rev. E, 50, R675-R678.

Pedersen, J. S., Posselt, D. \& Mortensen, K. (1990). J. Appl. Cryst. 23, 321-333.

Pine, D. J., Weitz, D. A., Chaikin, P. M. \& Herbolzheimer, E. (1988). Phys. Rev. Lett. 60, 1134-1137.

Pusey, P. N. \& van Megen, W. (1989). Physica A, 157, 705-741.

Ragnetti, M. \& Oberthür, R. C. (1986). Colloid Polym. Sci. 264, 3245.

Rojas-Ochoa, L., Romer, S., Scheffold, F. \& Schurtenberger, P. (2002). Phys. Rev. E, 65, 051403.
Romer, S., Scheffold, F. \& Schurtenberger, P. (2000). Phys. Rev. Lett. 85, 4980-4983.

Russel, W. B., Saville, D. A. \& Schowalter, W. R. (1989). Colloidal Dispersions. Cambridge University Press.

Scheffold, F., Skipetrov, S. E., Romer, S. \& Schurtenberger, P. (2001). Phys. Rev. E, 63, 061404.

Weitz, D. A. \& Pine, D. J. (1993). Dynamic Light Scattering, edited by W. Brown, pp. 652-721. Oxford: Clarendon Press.

Wignall, G. D. \& Bates, F. S. (1987). J. Appl. Cryst. 20, 28-40.

Xue, J. Z., Pine, D. J., Milner, S. T., Wu, X. L. \& Chaikin, P. M. (1992). Phys. Rev. A, 46, 6550-6563. 